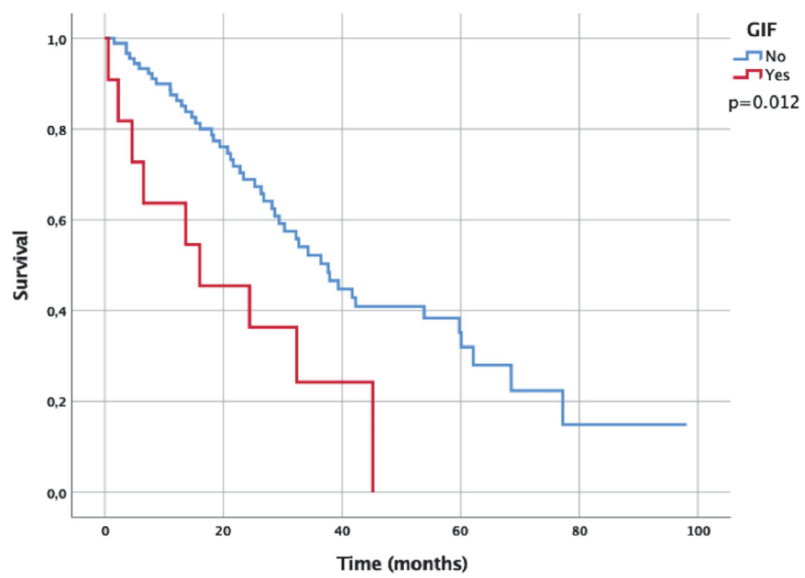

Abstract 270 Figure 2 Overall survival according to the existence or absence of GIF

Methodology We evaluated the clinicopathological features in patients with AOC and their association with GI. Data for 107 patients with AOC who underwent primary debulking surgery were analyzed retrospectively. Clinicopathological features, including demographic, surgical procedures and followup data, were analyzed in relation to GIF..

Result(s)* GIF was present in $11 \%$ of patients in the study, 5 $(4.5 \%)$ and $7(6.4 \%)$ of colorectal and small bowel origin, respectively. GIF was significantly associated with peritoneal cancer index $(\mathrm{PCI})>20$, more than 2 visceral resections, and multiple digestive resections. Overall and disease-free survival were also associated with GIF. Multivariate analysis identified partial bowel obstruction and operative bleeding as independent prognos- tic factors for survival. The presence of GIF is positively associated with poor prognosis in patients with AOC.

Conclusion* Given the importance of successful cytoreductive surgery in AOC, the assess- ment of the amount of tumor and the aggressiveness of the surgery to avoid the occurrence of GIF become a priority in patients with AOC.

\section{IMPROVEMENT OF COMPLETE CYTOREDUCTION FOR ADVANCED-STAGE OVARIAN CANCER WITH ADJUVANT USE OF THE PLASMAJET DEVICE. RESULTS OF A RCT}

${ }^{1,2} \mathrm{G}$ Nieuwenhuyzen-de Boer, ${ }^{3} \mathrm{~W}$ Hofhuis, ${ }^{4} \mathrm{~N}$ Reesink-Peters, ${ }^{5} \mathrm{~S}$ Willemsen, ${ }^{6} \mathrm{~A}$ Boere ${ }^{7}$ IG Schoots, ${ }^{8} \mathrm{~J}$ Piek, ${ }^{2} \mathrm{~L}$ Hofman, ${ }^{9} \mathrm{~J}$ Beltman, ${ }^{10} \mathrm{WJ}$ Van Driel, ${ }^{11} \mathrm{HMJ}$ Werner, ${ }^{12} \mathrm{M}$ Dorman ${ }^{13}$ L Haans, ${ }^{14} \mathrm{~A}$ Baalbergen, ${ }^{15}$ AMLDVan Haaften-de Jong, ${ }^{16} \mid$ Nedelcu, ${ }^{17}$ PC Ewing Graham, ${ }^{1} \mathrm{HJ}$ Van Beekhuizen. 'Erasmus University Medical Center, Gynaecologic Oncology, Rotterdam, Netherlands; '2Albert Schweitzer Hospital, Gynaecology, Dordrecht, Netherlands; ${ }^{3}$ Franciscus Gasthuis and Vlietland, Gynaecology, Rotterdam, Netherlands; ${ }^{4}$ MST, Gynaecology, Enschede, Netherlands; ${ }^{5}$ Erasmus University Medical Center, Epidemiology and Statistics, Rotterdam, Netherlands; ${ }^{6}$ Erasmus University Medical Center, Medical Oncology, Rotterdam, Netherlands; ' Erasmus University Medical Center, Radiology and Nuclear Medicine, Rotterdam, Netherlands; ${ }^{8}$ Catharina Ziekenhuis, Gynaecologic Oncology, Eindhoven, Netherlands; ${ }^{9}$ Leiden University Medical Center (LUMC), Gynaecologic Oncology, Leiden, Netherlands; ${ }^{10}$ The Netherlands Cancer Institute (NKI), Gynaecologic Oncology, Amsterdam, Netherlands; ${ }^{11}$ Academic Hospital Maastricht, Gynaecologic Oncology, Maastricht, Netherlands; ${ }^{12}$ Bravis Hospital, Gynaecology, Bergen op Zoom, Netherlands; ${ }^{13} \mathrm{HMC}$ Antoniushove, Gynaecology, Leidschendam, Netherlands; ${ }^{14}$ Reinier de Graaf Gasthuis, Gynaecology, Delft, Netherlands; ${ }^{15}$ Haga Hospital (Leyweg), Gynaecology, Den Haag, Netherlands; ${ }^{16}$ Groene Hart Hospital, Gynaecology, Gouda, Netherlands; ${ }^{17}$ Erasmus University Medical Center, Pathology, Rotterdam, Netherlands
Results of the PlaComOv-study, a randomized, controlled trial in the Netherlands

Introduction/Background* The most important prognostic factor for patients with advanced-stage ovarian cancer is complete cytoreductive surgery (CRS). Standard surgical treatment with electrosurgery cannot always result in complete CRS, especially when many small metastases are found on the mesentery and intestinal surface. We investigated whether adjuvant use of the PlasmaJet ${ }^{\circledR}$ Device will help increase the complete cytoreduction rate.

Methodology 327 patients with FIGO stage IIIB-IV epithelial ovarian cancer (EOC) who underwent primary or interval CRS were assigned to either surgery with PlasmaJet (intervention) or without PlasmaJet (control group).

Primary outcome was the percentage of complete CRS. Secondary outcomes were duration of surgery, blood loss, number of bowel resections and colostomies, hospitalization, 30 daymorbidity and quality of life (QoL).

Result(s)* Complete CRS was achieved in 119 patients $(75.8 \%)$ of the intervention group and in 115 patients (67.6\%) of the control group ( $\mathrm{P}=0.131)$.

A per-protocol analysis was performed in which data of 27 patients with unresectable disease were excluded, considering that the aim of the study was to examine the effectiveness of the use of the PlasmaJet in achieving CRS. Complete CRS was obtained in $85.6 \%$ in the intervention group and in $71.5 \%$ in the control group (absolute difference 14.1\%, 95\% confidence interval $[\mathrm{CI}], 0.047$ to $0.230 ; \mathrm{P}=0.005$ ).

The number of colostomies was lower in the intervention group (6.5\% versus $12.7 \%)$ but did not differ significantly $(\mathrm{P}=0.169)$. Patient-reported QoL six months after surgery differed between groups in favor of PlasmaJet surgery $(95 \% \mathrm{CI}$, 0.455 to $8.350 ; \mathrm{P}=0.029)$. Other secondary outcomes did not differ significantly.

Conclusion* The adjuvant use of the Plasmajet during CRS for advanced stage ovarian cancer resulted in a significant higher proportion of complete CRS in patients with resectable disease and a higher QoL six months after surgery. (Funded by ZonMw, Trial Register NL62035.078.17.)

\section{FEATURE SELECTION FOR TWO-YEAR PROGNOSIS IN ADVANCED STAGE HIGH GRADE SEROUS OVARIAN CANCER USING MACHINE LEARNING METHODS}

${ }^{1} \mathrm{~A}$ Laios ${ }^{*},{ }^{2} \mathrm{~A}$ Katsenou, ${ }^{3} \mathrm{Y}$ Tan, ${ }^{3} \mathrm{M}$ Otify, ${ }^{4} \mathrm{R}$ Hutson, ${ }^{1} \mathrm{~A}$ Thangavelu, ${ }^{4} \mathrm{~T}$ Broadhead, ${ }^{1} \mathrm{G}$ Theophilou, ${ }^{1,5} \mathrm{D}$ Nugent, ${ }^{1} \mathrm{D}$ Dejong. 'St James's University Hospital, Gynaecologic Oncology, Leeds, UK; ${ }^{2}$ Visual Inforamtion Lab, University of Bristol, Electrical and Electronic Engineering, Bristol, UK; ${ }^{3}$ St James's University Hospital, Gynaecologic Oncology, Leeds; ${ }^{4}$ St James's University Hospital, Gynaecologic Oncology, Leeds, UK; ${ }^{1}$ St James's University Hospital, Gynaecologic Oncology, Leeds, UK

\subsection{6/ijgc-2021-ESG0.376}

Introduction/Background* The prognosis of advanced stage high grade serous ovarian cancer patients (HGSOC) is multifactorial, and could be accurately predicted by using Machine Learning (ML) algorithms. We designed a study to support the feature selection of selected clinical variables to define their relative survival impact on two-year prognosis prediction in HGSOC patients, who received surgical treatment.

Methodology This was a retrospective analysis of 209 FIGO stage III-IV HGSOC women, who were scheduled for cytoreductive surgery in SJUH, Leeds between Jan 2015 to Dec 2018 with curative or life-prolonging intent. The two-year 
Two-year progression-free-survival Two-year overall-survival
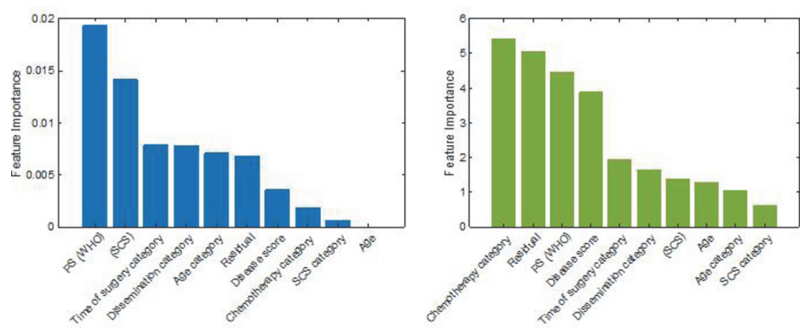

Abstract 282 Figure 1

prognosis estimation was formulated as a binary classification problem. Dataset was split into training (80\%) and test (20\%) cohorts with repeated random sampling until there was no significant difference $(p=0.20)$ between the two cohorts. A ten-fold cross-validation was applied. Various state-of-the-art supervised ML classifiers were tested, including Support-Vector-Machines (SVMs), K-Nearest Neighbors (KNNs), Ensemble Classifiers, and Naïve Bayes, based on a set of performance metrics. These results were directly compared to conventional Logistic Regression (LR). For feature selection, multivariate feature ranking using the MRMR method was carried out.

Result(s)* Two hundred nine patients were identified. The model's mean prediction accuracy reached $73 \%$. We demonstrated that SVM and Ensemble Discriminant algorithms outperformed Logistic Regression in accuracy indices. The probability of achieving a cancer-free state was maximized with a combination of primary cytoreduction, good performance status, and maximal surgical effort (AUC 0.63). Standard chemotherapy, performance status, tumor load, and residual disease were consistently predictive of the two-year overall survival (AUC 0.63-0.66) (figure 1). The model recall and precision were greater than $80 \%$.

Conclusion* Appropriate feature selection is required when building a HGSOC model for two-year prognosis prediction. For HGSOC prognosis, one should consider not only the patient's disease burden but also their overall medical status and ability to undergo extensive surgery, resulting in survival benefits alongside with standard chemotherapy.

\section{ANAPLASTIC MURAL NODULES WITHIN MUCINOUS OVARIAN CARCINOMA, A CASE SERIES ASSESSING TREATMENTS AND OUTCOMES}

L Baxter*, RK Ali Mohan, C Ayres, P Cohen, Y Leung. King Edward Memorial Hospital, Subiaco, Australia

\subsection{6/ijgc-2021-ESGO.377}

Introduction/Background* Mucinous ovarian tumours account for $\sim 10 \%$ of primary ovarian neoplasia and a generally diagnosed at an early stage with relatively favourable oncological outcomes. However those possessing anaplastic carcinoma within mural nodules rarer still and carry with them a poor prognosis and lack of consensus regarding their optimal treatment regime. Whilst a great deal of research has been published focusing on their histopathological and immunohistochemical characteristics, there is limited evidence nor consensus regarding their ideal adjuvant treatment regimes. This study sought to combine cases from our unit with a meta-analysis of cases in the literature to provide insight into current treatment regimes and outcomes.

Methodology A systematic review was conducted of the English language literature to identify articles published regarding outcomes and treatment modalities of patients having anaplastic carcinoma foci within mucinous ovarian tumours. References of these articles were reviewed to identify all possible cases in the literature. Where treatment regimes were not listed in the publications the contact author was reached for comment. These cases were then combined with 7 cases from our own institution for a multivariate and survival analysis.

Result(s)* A total of 66 cases were identified in the literature. Average age 43.7 (median 40.5), range 15-74yo. 83\% of patients underwent a total abdominal hysterectomy, $17 \%$ of patients did not under go a hysterectomy, with the remaining 2 cases having been done laparoscopically. $70 \%$ of patients underwent a BSO, the remainder a USO. $50 \%$ of cases were FIGO stage IA1 at the time of diagnosis, of these $16 \%$ died during follow up with 3 of those dying within 12 months of diagnosis.

Conclusion* Anaplastic mural nodules arising on a background of mucinous ovarian carcinoma are associated with heterogenous outcomes when considering progression free survival and overall survival. Their treatment within the literature is highly variable, particularly regarding adjuvant therapy. Patients with improved overall survival and progression free survival were more likely to be lower stage and have a smaller adnexal mass at diagnosis.

\section{CAN HE4 REPLACE CA 125 AS A BIOMARKER IN OVARIAN CANCER?}

A Ranjan, H Dubey, P Tanwar. AllMS, Delhi, Lab Oncology, Delhi, India

\subsection{6/ijgc-2021-ESG0.378}

Introduction/Background* Ovarian cancer is currently diagnosed using CAl25, which has a number of fallacies. Recently HE4 (human epididymis protein) is an emerging biomarker. It has higher potency to differentiate benign tumours from malignant one.

Methodology We studied 123 cases of ovarian cancer confirmed by histopathological examination. Whole blood samples were collected at the time of diagnosis prior to therapy (chemotherapy or surgery). We tested them for serum level of CA 125 \& HE4. Cut off values for HE4 and CA125 were <57.6 $\mathrm{pmol} / \mathrm{L}$ and $<39.6 \mathrm{U} / \mathrm{mL}$ respectively. Cut off values were calculated by ROC Curve analysis.

Result(s)* Total 123 cases were evaluated for serum level of HE4 \& CA125 prior to therapy. Results are being displayed in table 1 .

Out of 123 cases 38 showed CA 125 values negative, whereas the same was 14 for HE4, indicating a better diagnostic performance by HE4. As studied by Drapkin et al 2005, serum level of HE4 will not be raised in 50\% cases of clear-cell variant and almost all cases of mucinous tumors. It is positive in 93\% cases of serous tumors and almost all cases of endometrioid tumors. It will not be raised in benign ovarian cysts.

Among premenopausal cases (42/123), 10 showed CA 125 negative where as HE4 was negative in $7 / 42$ cases. Out of 10 CA 125 negative cases, 5 were HE4 positive. Out of HE4 